\title{
Prognostic role of SPARC expression in gastric cancer: a meta-analysis
}

Zishu Wang ${ }^{1}$, Bo Hao ${ }^{2}$, Yan Yang ${ }^{1}$, Rui Wang ${ }^{1}$, Yumei $\mathrm{Li}^{1}$, Qiong Wu

\author{
${ }^{1}$ Department of Medical Oncology, First Affiliated Hospital of Bengbu Medical College, \\ Bengbu, Anhui, China \\ 2Department of Gastrointestinal Surgery, First Affiliated Hospital of Bengbu Medical \\ College, Bengbu, Anhui, China
}

Submitted: 29 October 2013

Accepted: 5 January 2014

Arch Med Sci 2014; 10, 5: 863-869

DOI: 10.5114/aoms.2014.46207

Copyright $\odot 2014$ Termedia \& Banach

\section{Abstract}

Introduction: Secreted protein acidic and rich in cysteine (SPARC) is involved in regulating cell adhesion, proliferation, migration, and tissue remodeling. We performed a meta-analysis to evaluate the association between SPARC expression and the clinicopathologic features and outcomes of gastric cancer patients.

Material and methods: Publications that assessed the clinical or prognostic significance of SPARC in gastric cancer up to October 2013 were identified. A meta-analysis was performed to clarify the association between SPARC expression and clinical outcomes.

Results: Ten studies, including 1417 cases, met the inclusion criteria. The data were analyzed and the results show that SPARC is not significantly associated with the depth of gastric cancer invasion (odds ratio $(O R)=1.17$, $95 \%$ confidence interval $(\mathrm{Cl}): 0.60-2.29, Z=0.47, p=0.64)$ or tumor differentiation $(\mathrm{OR}=0.59,95 \% \mathrm{Cl}: 0.22-1.58, Z=1.06, p=0.29)$. Moreover, SPARC was not significantly correlated with lymph node metastasis $(O R=0.72$, $95 \% \mathrm{Cl}: 0.37-1.41, Z=0.96, p=0.34)$. However, SPARC overexpression was highly correlated with reduced overall survival (relative risk $(R R)=1.78$, $95 \% \mathrm{Cl}: 1.52-2.09, Z=7.10, p=0.43$ ).

Conclusions: The SPARC may play an important role in the progression of gastric cancer, and SPARC overexpression is closely correlated with poor patient survival. The SPARC is a potential clinical marker for the survival of gastric cancer patients; however, well-designed prospective studies are needed to confirm these findings.

Key words: secreted protein acidic and rich in cysteine, tumor progression, gastric cancer, prognosis.

\section{Introduction}

Gastric cancer is the most commonly diagnosed malignancy and is one of the most frequent causes of cancer mortality worldwide [1]. Although population screening programs in Japan have improved the early detection of gastric cancer and improved patient outcomes, the majority of gastric cancer patients outside Japan are still diagnosed at advanced stages. Advances in chemotherapy and surgical techniques have increased the survival rate of patients with gastric cancer, but the outcome of this common malignancy remains unsatisfactory because of poor understanding of the pathogenesis of gastric cancer and the lack of specific target gene

\author{
Corresponding author: \\ Dr. Qiong Wu \\ Department \\ of Medical Oncology \\ First Affiliated Hospital \\ of Bengbu Medical College \\ 287 Changhuai Road \\ Bengbu 233004, Anhui, China \\ Phone: +865523074480 \\ Fax: +865523074480 \\ E-mail: qiongwu63@126.com
}


therapy. The pathogenesis of gastric carcinomas is multifactorial, including genetic predisposition and environmental factors. Genetic predisposition is accompanied by several genetic alternations, including tumor suppressor genes, oncogenes, cell adhesion molecules, growth factors, and genetic stability [2]. Therefore, the molecular mechanisms involved in gastric cancer should be understood further and more prognostic markers should be identified to improve prognosis and to provide novel therapeutic targets.

Secreted protein acidic and rich in cysteine (SPARC), also known as osteonectin or BM-40, is a highly conserved multifunctional glycoprotein that belongs to the matricellular class of proteins [3]. Matricellular proteins function as adaptors that mediate cell-extracellular matrix (ECM) interactions and are expressed in tissues undergoing repair or remodeling. The SPARC is involved in regulating cell adhesion, proliferation, migration, and tissue remodeling and is expressed during cell development and processes that require extracellular matrix turnover, such as wound healing and tumor progression $[4,5]$. The SPARC expression is altered in many cancers. Increased SPARC expression has been observed in melanoma, glioma, colorectal, and breast carcinomas compared to their respective normal tissues [6]. In these tumors, high levels of SPARC are often correlated with enhanced invasion and metastasis [7]. By contrast, increased SPARC expression in ovarian carcinoma cells causes increased tumor cell apoptosis and is inversely correlated with tumor progression in vivo [8]. In pancreatic tumors, SPARC expression in cancer cells is limited by promoter hypermethylation, whereas infiltrating stromal cells exhibit increased SPARC expression [9]. Thus, the context of SPARC expression in the microenvironment is critical for understanding its influence on tumor growth and progression. Many studies have recently reported the correlation between SPARC expression and gastric cancer, but their results were inconclusive partially because of the relatively small sample size in each published study. Therefore, we performed a meta-analysis to derive a more precise estimate of the association between SPARC expression and gastric cancer.

\section{Material and methods}

\section{Publication search}

We performed a comprehensive search of the literature for abstracts of studies involving human subjects to identify articles regarding the prognostic role of SPARC expression in gastric cancer. Articles from January 1995 to October 2013 in the Medline, Embase, and Wanfang databases were searched using the following key words: "secreted protein, acidic and rich in cysteine", "SPARC", "osteonectin”, "BM-40”, "gastric carcinoma”, "gastric cancer", "overall survival”, and "OS". All searched studies were retrieved, and their bibliographies were checked for other relevant studies, which were manually searched to find additional eligible studies.

Information was carefully extracted independently from all eligible publications by two of the authors according to the inclusion criteria. Disagreements were resolved through consensus. The inclusion criteria were as follows: (1) articles dealing with SPARC expression and either the prognostic factors or overall survival (OS) of gastric cancer; (2) articles containing sufficient data that allow estimation of the odds ratio (OR) or relative risk (RR) of OS; (3) articles reported in English or Chinese; and (4) articles published as original research. Reviews, comments, duplicated studies, and articles unrelated to our analysis were excluded. Studies with follow-up periods less than 2 years were also excluded.

The following information was extracted from the included papers: author, publication year, patient's racial/ethnic background, tumor stage, number of patients, cell type of SPARC expression, research technique used, and definition of SPARC high expression. Two major groups were created according to the objectives. The first group clarified the association between SPARC expression and the clinicopathologic parameters, including depth of invasion, degree of differentiation, and lymph node involvement. The other group investigated the association between SPARC expression and OS.

\section{Statistical analysis}

The meta-analysis was performed as previously described [10]. For ease of analysis, the SPARC expression data and the clinicopathologic factors were combined into single categories: high SPARC expression and negative SPARC expression, and low SPARC expression; T1 and T2 stages; T3 and T4 stages; and well differentiated and moderately differentiated. The ORs with $95 \% \mathrm{Cl}$ were used to evaluate the association between SPARC expression and clinicopathologic factors such as tumor differentiation, lymph node involvement, and lymphatic invasion. The survival data were extracted using scanned images of published curves. Differences in SPARC expression and OS were quantified using RR with $95 \% \mathrm{Cl}$. Heterogeneity across studies was evaluated using a $\mathrm{Q}$ test and $p$ values. The ORs and RRs were calculated using a random effects model when the $p$ values were less than 0.05 . Otherwise, a fixed effects model was used. A Begg and Egger funnel plot was used to assess publication bias. Statistical analyses were performed 
using the Review Manager software. The $p$ values were two-sided, with significance at $p<0.05$.

\section{Results}

\section{Description of studies}

The results of the search strategy for studies are summarized in Figure 1. Finally, a total of 10 publications met the criteria for this analysis [1120]. The total number of patients was 1417, ranging from 43 to 436 patients per study. The main characteristics of the eligible studies are summarized in Table I. Seven articles dealt with clinicopathologic factors, 6 studies determined the OS, and only three studies reported the association between SPARC expression and clinicopathologic factors without OS analysis. The studies mainly used two methods to evaluate SPARC expression in gastric cancer specimens: immunohistochemistry (IHC) and reverse transcription PCR (RT-PCR).

\section{Correlation of SPARC expression with clinicopathologic parameters}

Six studies assessed the relationship between SPARC phenotype and depth of invasion (Figure 2). The pooled OR was 1.17 (95\% Cl: 0.60-2.29, Z = $0.47, p=0.64$ random effects). The SPARC expression was not significantly correlated with depth of invasion (Figure 2). The SPARC expression was not significantly correlated with clinicopathologic parameters such as tumor differentiation (pooled $\mathrm{OR}=0.59,95 \% \mathrm{Cl}: 0.22-1.58, Z=1.06, p=0.29$ random effects) and lymph node metastasis (OR = $0.72,95 \% \mathrm{Cl}: 0.37-1.41, Z=0.96, p=0.34$ random effects) (Figures 3 and 4). Moreover, Egger's test indicated that these clinicopathologic parameters showed no significant publication bias (Figure 5).

\section{Effect of SPARC expression on overall survival of gastric cancer}

A meta-analysis was performed on six studies that investigated the association between SPARC expression and OS. The pooled RR was calculated using the methods described above. The test for heterogeneity was significant $(p<0.0001)$; thus, a random effects model was used to calculate the RR. The presence of SPARC expression was correlated with poor OS (pooled $R R=1.78$, 95\% Cl: 1.52-2.09, $Z=7.10, p=0.43$ ) (Figure 6). This finding indicates that SPARC is an independent prognostic factor for gastric cancer. No significant publication bias was detected (Figure 5).

\section{Discussion}

To the best of our knowledge, the present meta-analysis is the first English language study to systematically determine the association between

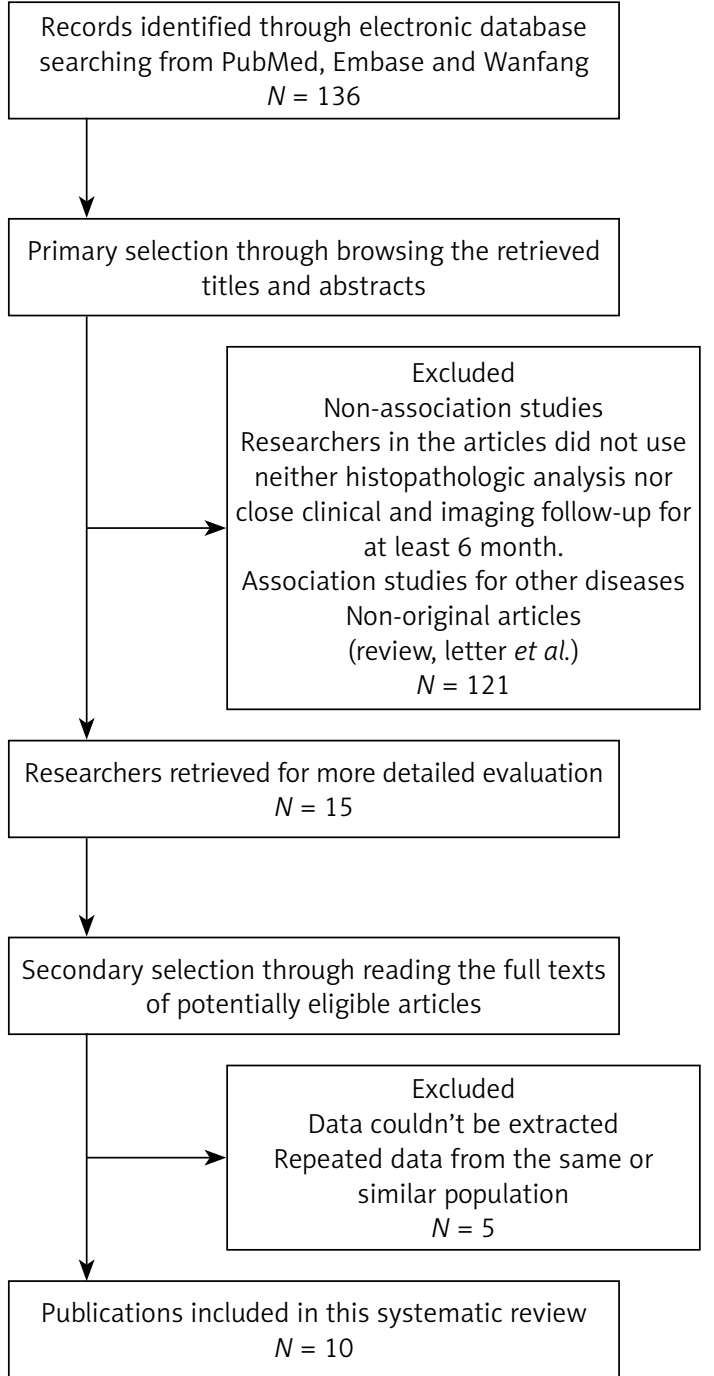

Figure 1. Methodological flow diagram of the systematic review

SPARC expression and gastric cancer survival. The prognostic and predictive significance of SPARC expression in gastric cancer is controversial; for example, Zhao et al. [13] found that expression of SPARC in gastric cancer was significantly associated with lymph node and distant metastasis and poor prognosis. However, Wang et al. [19] reported that SPARC inhibited the development and progression of gastric cancer; thus, a quantitative meta-analysis of study outcomes is warranted. Our analysis indicated that SPARC expression is significantly associated with OS, which indicates that SPARC is a potential marker for poor gastric cancer prognosis.

Recent studies have shown that SPARC modulates the proliferation, apoptosis, invasion, and angiogenesis of different types of cancers; however, the underlying mechanism remains unknown. Some articles have revealed that SPARC is upregulated in gastric cancer tissues compared with that in normal gastric tissues $[13,17]$. In our study, the 


\begin{tabular}{|c|c|c|c|c|c|c|c|c|c|c|}
\hline 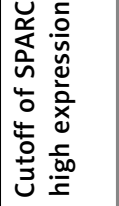 & 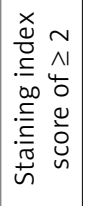 & 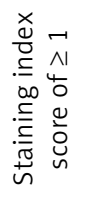 & 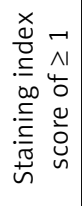 & 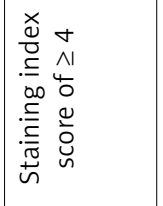 & 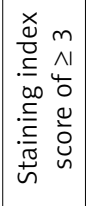 & 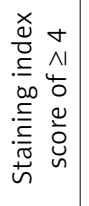 & 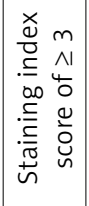 & 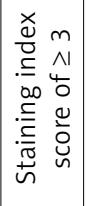 & 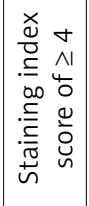 & z \\
\hline 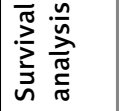 & $\tilde{o}$ & 之 & กิ & ก & 乞 & 之े & $\tilde{o}$ & 之 & z & 乞ั \\
\hline 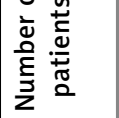 & $\stackrel{m}{\forall}$ & $\stackrel{\infty}{\stackrel{\sim}{\sim}}$ & $\underset{\beth}{\stackrel{ \pm}{\prime}}$ & $\begin{array}{l}\stackrel{\infty}{\vartheta} \\
\forall\end{array}$ & ৪ & $\infty$ & 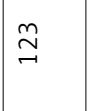 & $\infty$ & $\infty$ & $\stackrel{\widehat{N}}{ }$ \\
\hline 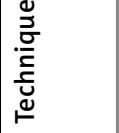 & 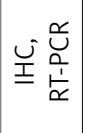 & $\underline{\underline{\underline{x}}}$ & 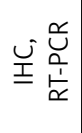 & 喜 & 呈 & 呈 & 堊 & $\underline{\underline{\underline{x}}}$ & $\underline{\underline{\underline{I}}}$ & 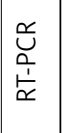 \\
\hline 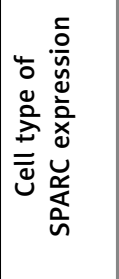 & 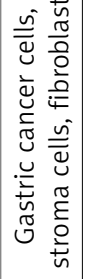 & 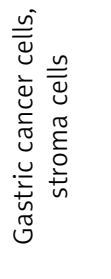 & 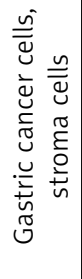 & 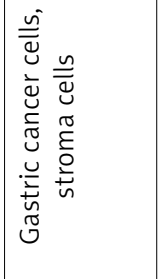 & z & 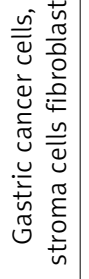 & 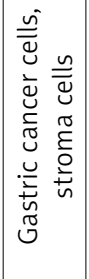 & 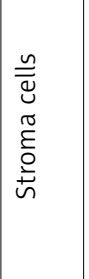 & 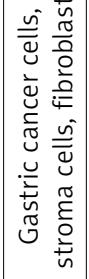 & ₹ \\
\hline 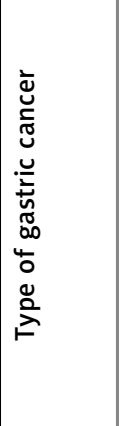 & 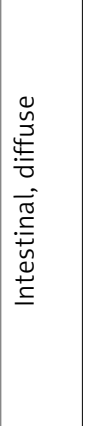 & 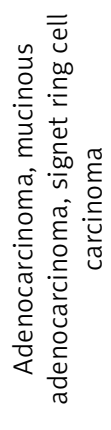 & 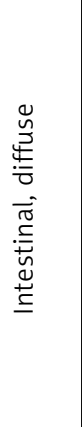 & 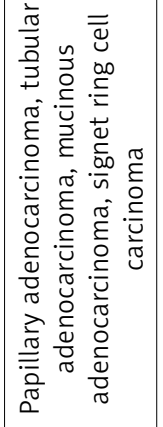 & 2 & 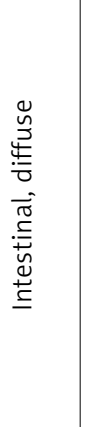 & 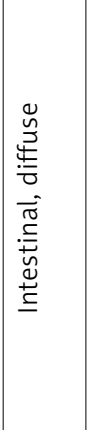 & 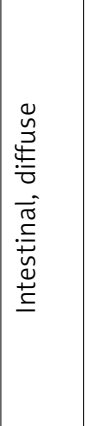 & 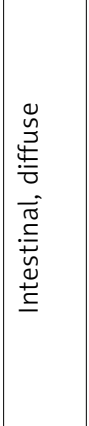 & 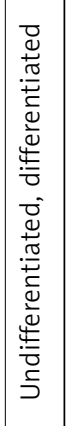 \\
\hline 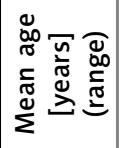 & $\begin{array}{l}\widehat{\widehat{m}} \\
0 \\
\hat{\omega} \\
\tilde{m} \\
\vec{b}\end{array}$ & $\begin{array}{l}\text { à } \\
\text { a } \\
\text { in }\end{array}$ & 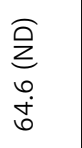 & $\begin{array}{l}\bar{a} \\
\hat{0} \\
0 \\
0 \\
0 \\
0\end{array}$ & $\begin{array}{l}\underset{n}{n} \\
i \\
\stackrel{n}{\simeq} \\
\infty \\
i n\end{array}$ & $\begin{array}{l}\widehat{\widehat{m}} \\
0 \\
\omega \\
\tilde{m} \\
\overrightarrow{0} \\
\overrightarrow{0}\end{array}$ & 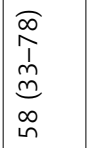 & 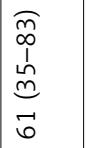 & $\begin{array}{l}o \\
\infty \\
o \\
\sim \\
o \\
o \\
o \\
i n\end{array}$ & 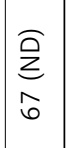 \\
\hline 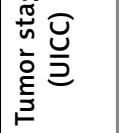 & $\stackrel{\geq}{I}$ & $\underset{I}{\geq}$ & $\underset{I}{\geq}$ & $\vec{I}$ & $\stackrel{\geq}{I}$ & $\stackrel{\geq}{I}$ & $\underset{I}{\geq}$ & $\underset{I}{\geq}$ & $\stackrel{\geq}{I}$ & $\stackrel{\geq}{I}$ \\
\hline ঠ্ & 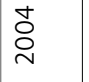 & ¿্ণ & ஓे & $\stackrel{\stackrel{\circ}{\sim}}{\stackrel{i}{N}}$ & $\vec{D}$ & $\vec{\sim}$ & $\stackrel{\sim}{\stackrel{\sim}{\sim}}$ & $\underset{\sim}{\sim}$ & $\stackrel{\sim}{\stackrel{\sim}{\sim}}$ & $\underset{\sim}{n}$ \\
\hline 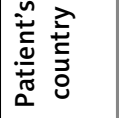 & 茎 & 胥 & 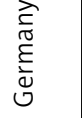 & 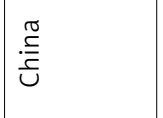 & 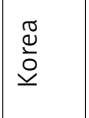 & 胥 & 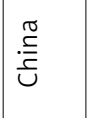 & : & 疍 & 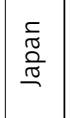 \\
\hline 咅 & 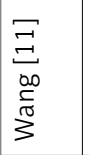 & $\underset{\Xi}{\Xi}$ & 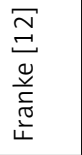 & $\begin{array}{l}\bar{m} \\
\stackrel{\Xi}{0} \\
\frac{\pi}{N} \\
\frac{d}{N}\end{array}$ & 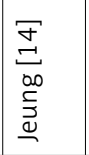 & $\begin{array}{l}\sqrt[n]{n} \\
\stackrel{5}{ \pm} \\
\stackrel{5}{I} \\
\text { I }\end{array}$ & $\begin{array}{l}\bar{\Xi} \\
\stackrel{\Xi}{0} \\
\alpha \\
\bar{o} \\
\Delta\end{array}$ & $\begin{array}{l}\frac{\sigma}{\Xi} \\
\stackrel{0}{00} \\
\frac{\pi}{\pi} \\
3\end{array}$ & 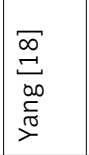 & 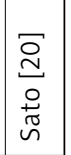 \\
\hline
\end{tabular}




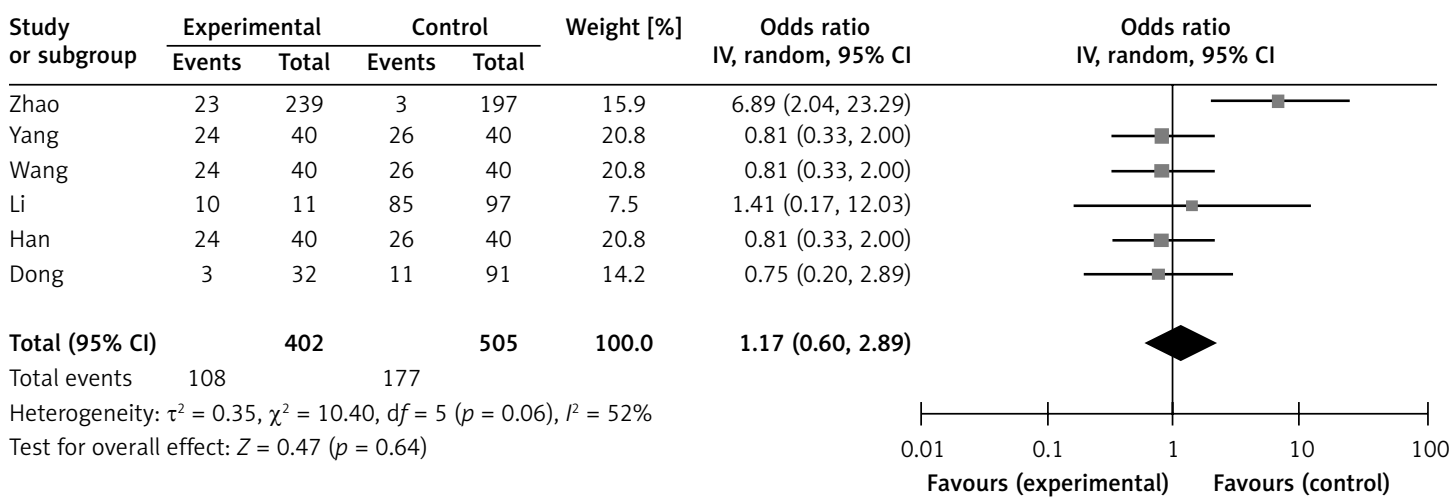

Figure 2. Forest plot of odds ratios (ORs) for the association of SPARC expression with depth of invasion

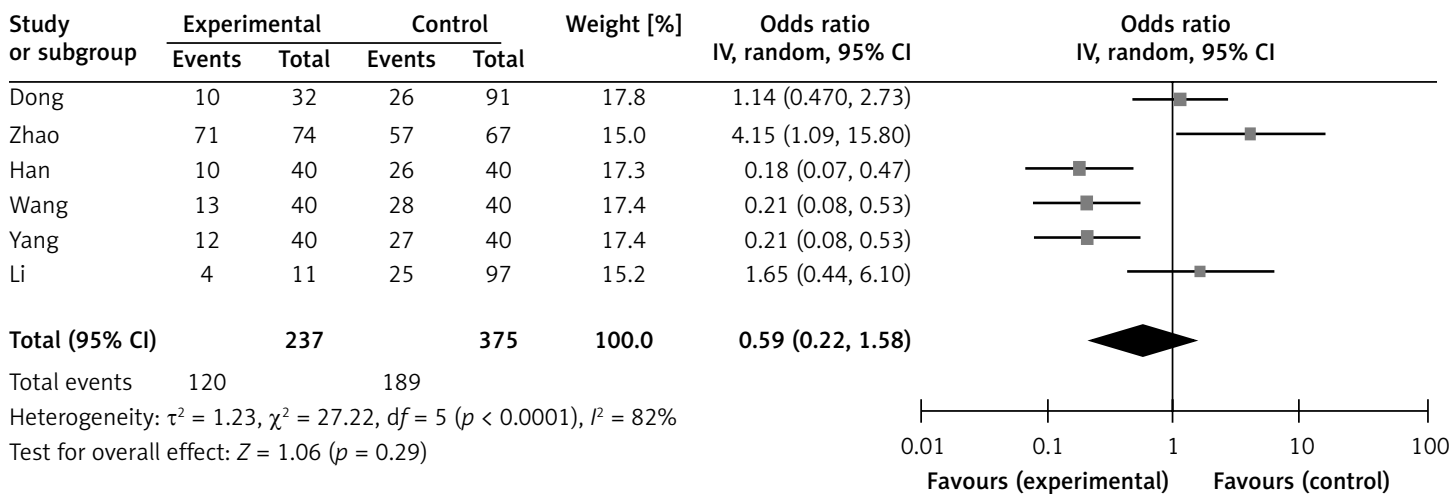

Figure 3. Forest plot of ORs for the association of SPARC expression with tumor differentiation

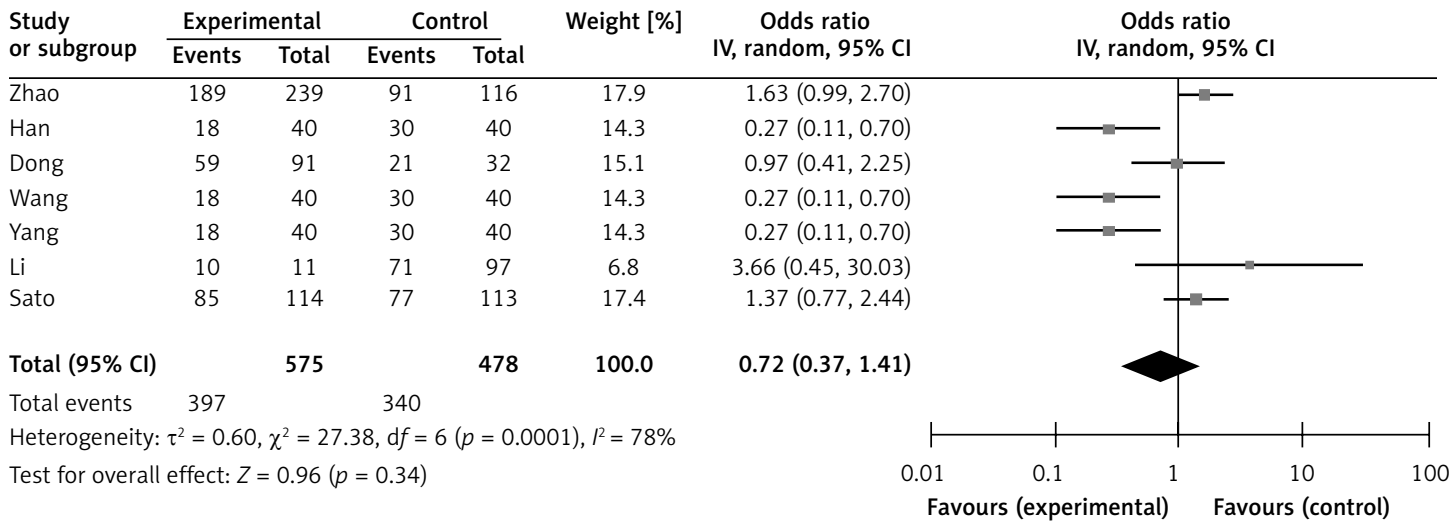

Figure 4. Forest plot of ORs for the association of SPARC expression with lymph node metastasis

data show that SPARC is not correlated with lymph node metastasis or degree of tumor differentiation. Although deeper invasion was more common in cases with high SPARC expression than in those with negative or low SPARC expression, the differences were not statistically significant. Some studies reported a significant association between SPARC expression and gastric cancer [11-13], and that SPARC might be considered a new marker for the staging and management of gastric cancer. Our meta-analysis suggests that patients with high expression of SPARC have worse prognosis than those with low expression of SPARC, which suggests that SPARC may be useful as a prognostic indicator. However, our results should be interpreted with extreme caution. Several factors that may influence the conclusion of each included report should also be considered, such as sample size, immunohistochemical staining patterns (membrane versus cytoplasm), and the choice of cutoff scores for positive staining or staining intensity.

This meta-analysis has some limitations. First, the number of included studies is relatively small, with only 1417 cases. Gastric cancer patients received different treatments (perioperative adjuvant therapy or curative surgical resection) and had 

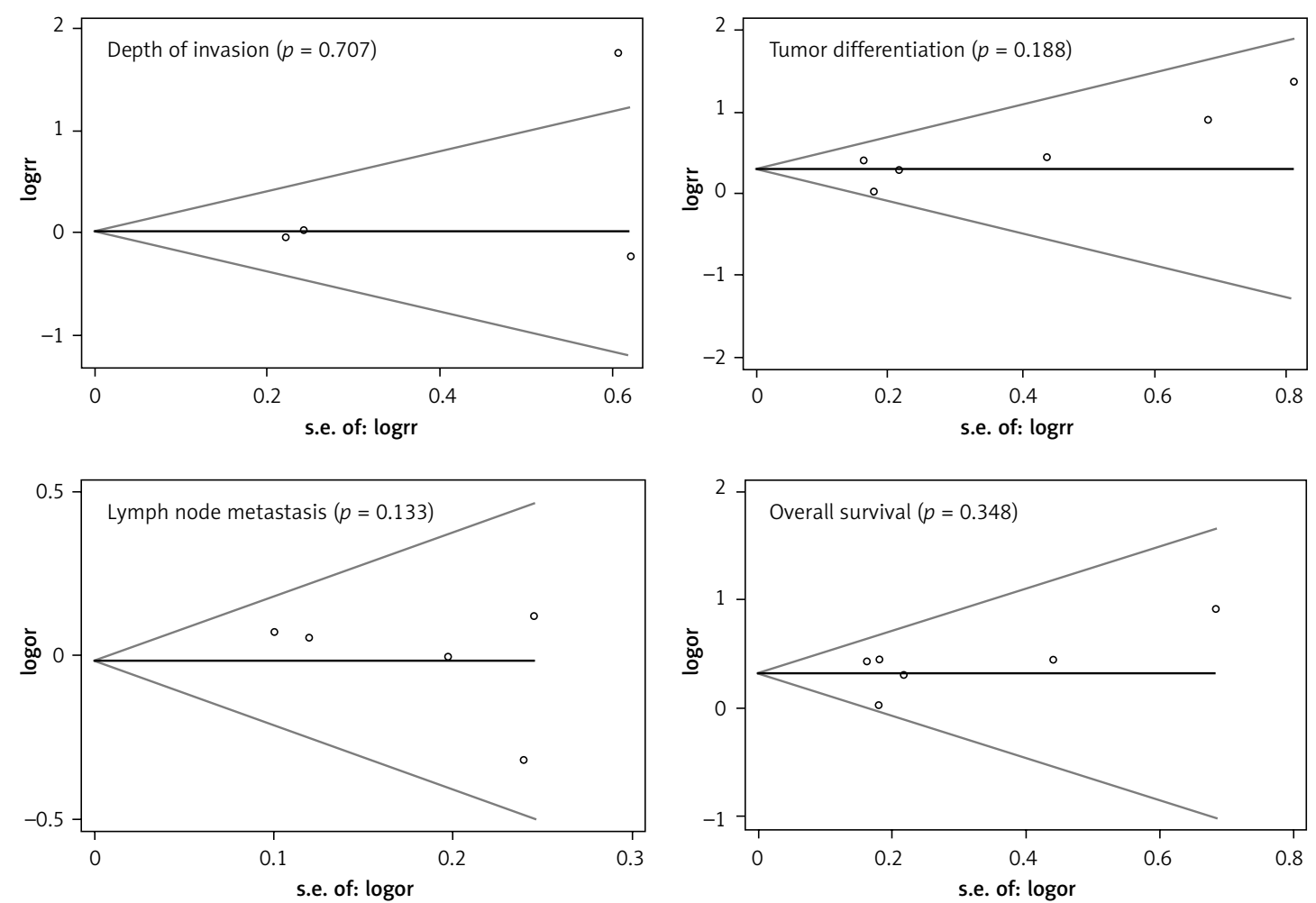

Figure 5. Begg's funnel plots for assessing the publication bias risk

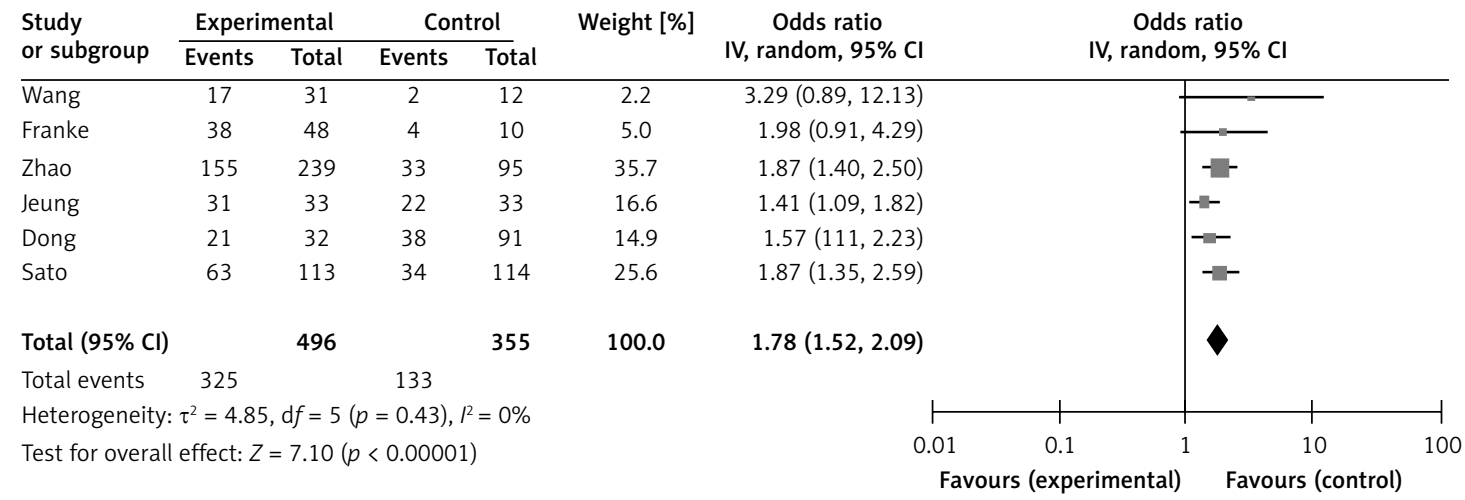

Figure 6. Forest plot of relative risk (RR) for the association of SPARC expression with overall survival (OS)

varying preoperative TNM categories and histologic types. We were unable to assess the potential confounding variables in the individual studies. Second, although immunohistochemistry was the most commonly applied method for detecting SPARC in situ, the RT-PCR method was also used to evaluate the expression levels SPARC in tumor tissues. Therefore, the impact of SPARC expression on prognosis in gastric cancer should be further validated. Third, nine of the ten studies in this meta-analysis evaluated patients from Asian populations; thus, more studies should include populations from different ethnicities and regions to confirm our findings. Moreover, the cutoff values were defined differently in the included studies, causing between-study heterogeneity. Thus, we adopted a random effects model and performed subgroup sensitivity analyses to compensate for this limitation.

In conclusion, our results indicate that SPARC expression is not associated with the common clinicopathologic parameters of gastric cancer, such as depth of invasion, tumor differentiation, and lymph node metastasis. However, high SPARC expression is associated with worse outcomes than negative and low SPARC expression, and SPARC is an independent indicator of reduced survival. Further studies on SPARC and its potential as a clinical marker for gastric cancer prognosis are warranted.

\section{Acknowledgments}

Bo Hao is co-first author. 


\section{References}

1. Kamangar F, Dores GM, Anderson WF. Patterns of cancer incidence, mortality, and prevalence across five continents: defining priorities to reduce cancer disparities in different geographic regions of the world. J Clin Oncol 2006; 24: 2137-50.

2. Ajani JA. Evolving chemotherapy for advanced gastric cancer. Oncologist 2005; 10 Suppl. 3: 49-58.

3. Brekken RA, Sage EH. SPARC, a matricellular protein: at the crossroads of cell-matrix communication: [Matrix Biology (2000) 569-580]. Matrix Biology 2001; 19: 815-27.

4. Funk SE, Sage EH. The Ca2 (+)-binding glycoprotein SPARC modulates cell cycle progression in bovine aortic endothelial cells. Proc Nat Acad Sci 1991; 88: 2648-52.

5. Lane T, Sage E. The biology of SPARC, a protein that modulates cell-matrix interactions. FASEB J 1994; 8: 163-73.

6. Framson PE, Sage EH. SPARC and tumor growth: where the seed meets the soil? J Cell Biochem 2004; 92: 679-90.

7. Arnold S, Mira E, Muneer S, et al. Forced expression of MMP9 rescues the loss of angiogenesis and abrogates metastasis of pancreatic tumors triggered by the $a b-$ sence of host SPARC. Exp Biol Med 2008; 233: 860-73.

8. Brown TJ, Shaw PA, Karp X, Huynh MH, Begley H, Ringuette MJ. Activation of SPARC expression in reactive stroma associated with human epithelial ovarian cancer. Gynecol Oncol 1999; 75: 25-33.

9. Sato N, Fukushima N, Maehara N, et al. SPARC/osteonectin is a frequent target for aberrant methylation in pancreatic adenocarcinoma and a mediator of tumorstromal interactions. Oncogene 2003; 22: 5021-30.

10. Zhou L, Jiang Y, Yan T, et al. The prognostic role of cancer stem cells in breast cancer: a meta-analysis of published literatures. Breast Cancer Res Treat 2010; 122: 795-801.

11. Wang CS, Lin KH, Chen SL, Chan YF, Hsueh S. Overexpression of SPARC gene in human gastric carcinoma and its clinic - pathologic significance. Br J Cancer 2004; 91: 1924-30.

12. Franke K, Carl-McGrath S, Röhl FW, et al. Differential expression of SPARC in intestinal-type gastric cancer correlates with tumor progression and nodal spread. Translat Oncol 2009; 2: 310

13. Zhao ZS, Wang YY, Chu YQ, Ye ZY, Tao HQ. SPARC is associated with gastric cancer progression and poor survival of patients. Clin Cancer Res 2010; 16: 260-8.

14. Jeung HC, Rha SY, Im CK, et al. A randomized phase 2 study of docetaxel and S-1 versus docetaxel and cisplatin in advanced gastric cancer with an evaluation of SPARC expression for personalized therapy. Cancer 2011; 117: 2050-7.

15. Han W, Yang ML, Zhai LL, et al. Relationship between expression of SPARC and TGF-beta1 with cancer cells proliferation in gastric cancer. J Clin Exp Pathol 2011; 27: 594-7.

16. Dong MX. Expression of SPARC in gastric carcinoma and its clinical significance. Qilu Med J 2011; 4: 105-7.

17. Li Y, Wu JF, Zhang H, et al. Expression of SPARC in gastric cancer. Acta Universitatis Medicinalis Anhui 2006; 41: 626-9.

18. Yang ML, Zhai LL, Ma LH, et al. Relationship between expression of SPARC and VEGF with tumor angiogenesis in gastric carcinoma. J Diag Pathol 2012; 19: 52-5.

19. Wang L, Yang M, Shan L, et al. The role of SPARC protein expression in the progress of gastric cancer. Pathol Oncol Res 2012; 18: 697-702.

20. Sato T, Oshima T, Yamamoto $\mathrm{N}$, et al. Clinical significance of SPARC gene expression in patients with gastric cancer. J Surg Oncol 2013; 108: 364-8. 